\title{
Comparison of the $\mathrm{H} \alpha$ circumstellar disks in $\mathrm{Be} / \mathrm{X}$-ray binaries and Be stars
}

\author{
R. K. Zamanov ${ }^{1}$, P. Reig ${ }^{2,3}$, J. Martí ${ }^{4}$, M. J. Coe $^{5}$, J. Fabregat ${ }^{6}$, N. A. Tomov ${ }^{1}$, and T. Valchev ${ }^{7}$ \\ 1 Institute of Astronomy, Bulgarian Academy of Sciences and Isaac Newton Institute of Chile, Bulgarian Branch, \\ National Astronomical Observatory Rozhen, PO Box 136, 4700 Smolyan, Bulgaria \\ 2 Foundation for Research and Technology-Hellas, 71110 Heraklion, Crete, Greece \\ 3 Physics Department, University of Crete, 71003 Heraklion, Crete, Greece \\ 4 Departamento de Física, EPS, Universidad de Jaén, C/ Virgen de la Cabeza, 2, 23071 Jaén, Spain \\ 5 Physics and Astronomy Department, Southampton University, Southampton, S017 1BJ, UK \\ 6 Departamento de Astronomía, Universidad de Valencia, 46100 Burjassot, Valencia, Spain \\ 7 National Astronomical Observatory Rozhen, PO Box 136, 4700 Smolyan, Bulgaria
}

Received 2 June 2000 / Accepted 14 December 2000

\begin{abstract}
We present a comparative study of the circumstellar disks in Be/X-ray binaries and isolated Be stars based upon the $\mathrm{H} \alpha$ emission line. From this comparison it follows that the overall structure of the disks in the $\mathrm{Be} / \mathrm{X}$-ray binaries is similar to the disks of other Be stars, i.e. they are axisymmetric and rotationally supported. The factors for the line broadening (rotation and temperature) in the disks of the Be stars and the Be/X-ray binaries seem to be identical. However, we do detect some intriguing differences between the envelopes. On average, the circumstellar disks of the Be/X-ray binaries are twice as dense as the disks of the isolated Be stars. The different distribution of the Be/X-ray binaries and the Be stars seen in the full width half maximum versus peak separation diagram indicates that the disks in Be/X-ray binaries have on average a smaller size, probably truncated by the compact object.
\end{abstract}

Key words. stars: emission - line, Be - circumstellar matter - binaries: close - X-rays: stars

\section{Introduction}

Be stars are early type non-supergiant stars surrounded by a circumstellar envelope with disk-like geometry. The circumstellar disk is visible in the Balmer (and sometimes other) line emission and also gives rise to a strong infrared excess. The physical mechanism of the disk formation is not well understood, but it is generally believed that the fast rotation of the central star plays an important role. The Be phenomenon is present in both isolated B type stars and X-ray binaries with massive B type companions. The Be/X-ray binaries represent the major subclass of massive $\mathrm{X}$-ray binaries, in which the $\mathrm{X}$-ray emission is due to the interaction of the compact object (usually a neutron star) with the wind of the Be star.

In this paper, we explore the existence of significative differences between the Be disks in both kinds of systems. The black hole, or neutron star, companion in an X-ray binary may be affecting the Be disk structure. Is there any observational evidence? Can we see the signature of the compact companion in the Be emission line profiles?

Send offprint requests to: R. K. Zamanov, e-mail: rozhen@mbox.digsys.bg
Traditionally, the answer to this question has been negative, and it was believed that the presence of the compact object would not affect the dynamics of the Be disk. However, recent results seem to contradict this idea. Reig et al. (1997) found a relationship between the properties of the disk and the orbit of the neutron star. They reported that Be stars in X-ray binary systems have, on average, a lower $\mathrm{H} \alpha$ equivalent width $(E W)$ when compared to a complete set - those contained in the Bright Star Catalogue (Hoffleit \& Jascheck 1982) - of isolated Be stars. They also discovered a possible correlation between the maximum observed $E W(\mathrm{H} \alpha)$ and the orbital period of the system, which was explained by assuming that the compact object truncates the outer parts of the Be star's disk. The idea of disk truncation, together with disk warping has been used by Negueruela et al. (2000) to explain the X-ray outburst mechanism in the Be/X-ray system 4U 0115+63 (V635 Cas).

The solution to these questions is likely to be provided by a careful and exhaustive examination of high dispersion optical and infrared (IR) spectra at high signalto-noise ratio $(\mathrm{S} / \mathrm{N})$. The following sections are devoted to a comparative study of the circumstellar disks in the 
Table 1. H $\alpha$ line parameters from Southampton - Valencia data base

\begin{tabular}{|c|c|c|c|c|}
\hline $\begin{array}{c}\text { Date } \\
\text { (yyyymmdd) }\end{array}$ & $\begin{array}{c}-E W(\mathrm{H} \alpha) \\
(\AA)\end{array}$ & $\begin{array}{c}\Delta V \\
\left(\mathrm{~km} \mathrm{~s}^{-1}\right)\end{array}$ & $\begin{array}{l}F W H M \\
\left(\mathrm{~km} \mathrm{~s}^{-1}\right)\end{array}$ & $\begin{array}{c}\text { Disp. } \\
\AA_{\text {pix }}^{-1}\end{array}$ \\
\hline \multicolumn{5}{|c|}{ 4U1145-619/V801 Cen } \\
\hline 19850101 & 18.2 & 205 & 363 & 0.8 \\
\hline 19850102 & 17.7 & - & 368 & 0.8 \\
\hline 19850103 & 17.6 & - & 292 & 0.8 \\
\hline 19850105 & 16.3 & 205 & - & 0.8 \\
\hline 19930302 & 38.0 & - & 379 & 0.5 \\
\hline 19940307 & 45.0 & 98 & 420 & 0.5 \\
\hline 19940702 & 25.0 & - & 457 & 0.5 \\
\hline 19950819 & 27.0 & 216 & 370 & 0.5 \\
\hline 19960302 & 16.0 & 174 & 297 & 0.3 \\
\hline 19960404 & 14.0 & 227 & 288 & 0.5 \\
\hline \multicolumn{5}{|c|}{ A0535+26/V725 Tau } \\
\hline 19880310 & 14.0 & - & 462 & 2.6 \\
\hline 19901114 & 9.9 & 140 & 347 & 1.0 \\
\hline 19901227 & 8.6 & 167 & 384 & 0.5 \\
\hline 19910828 & 9.4 & 198 & 384 & 0.5 \\
\hline 19920818 & 7.4 & 218 & 343 & 0.8 \\
\hline 19931206 & 13.0 & - & 370 & 0.8 \\
\hline 19940325 & 9.0 & - & 343 & 1.3 \\
\hline 19940916 & 9.4 & 199 & 361 & 1.3 \\
\hline 19941110 & 12.0 & 228 & 393 & 0.8 \\
\hline 19950226 & 8.6 & 212 & 361 & 0.5 \\
\hline 19950806 & 8.0 & 230 & 402 & 0.4 \\
\hline 19951120 & 6.2 & 244 & 416 & 0.5 \\
\hline 19960228 & 7.3 & 233 & 416 & 0.4 \\
\hline \multicolumn{5}{|c|}{ V0332+53/BQ Cam } \\
\hline 19900128 & 4.5 & - & 224 & 0.3 \\
\hline 19900902 & 5.0 & - & 293 & 1.0 \\
\hline 19901114 & 5.7 & - & 297 & 1.0 \\
\hline 19910127 & 6.5 & - & 251 & 1.0 \\
\hline 19910828 & 4.6 & 143 & 274 & 0.5 \\
\hline 19911214 & 4.8 & 153 & 297 & 0.5 \\
\hline 19920818 & 3.1 & - & 265 & 0.8 \\
\hline 19921113 & 3.8 & - & 274 & 0.8 \\
\hline 19970814 & 4.2 & - & 280 & 0.4 \\
\hline 19971114 & 3.9 & 155 & 270 & 0.4 \\
\hline
\end{tabular}

"normal" Be stars and the Be/X-ray binaries based on the best available $\mathrm{H} \alpha$ emission line spectroscopy.

\section{Spectroscopic data}

In our comparative study we used the $\mathrm{H} \alpha$ equivalent width, full width at half maximum $(F W H M)$ and the distance between the peaks $(\Delta V)$. The data for the "normal" Be stars were taken from the high resolution, high S/N observations of Andrillat (1983); Hanuschik (1986); Hanuschik et al. (1988); Dachs et al. (1992); Slettebak et al. (1992). The optical counterparts of the Be/X-ray binaries have spectral classes in the range O8-B2, and represent a significantly different distribution from that of isolated Be stars (Negueruela 1998). But as far as we
Table 1. continued

\begin{tabular}{|c|c|c|c|c|}
\hline $\begin{array}{c}\text { Date } \\
\text { (yyyymmdd) }\end{array}$ & $\begin{array}{c}-E W(\mathrm{H} \alpha) \\
(\AA)\end{array}$ & $\begin{array}{c}\Delta V \\
\left(\mathrm{~km} \mathrm{~s}^{-1}\right)\end{array}$ & $\begin{array}{l}F W H M \\
\left(\mathrm{~km} \mathrm{~s}^{-1}\right)\end{array}$ & $\begin{array}{c}\text { Disp. } \\
\text { A pix }^{-1}\end{array}$ \\
\hline \multicolumn{5}{|c|}{ 4U0115+63/V635 Cas } \\
\hline 19900214 & 6.8 & 182 & 571 & 1.0 \\
\hline 19901227 & 4.5 & 400 & 603 & 0.5 \\
\hline 19910127 & 9.5 & - & 452 & 1.0 \\
\hline 19910828 & 3.9 & 453 & 640 & 0.5 \\
\hline 19911214 & 5.4 & 497 & 699 & 0.5 \\
\hline 19920805 & 4.1 & 425 & 608 & 1.2 \\
\hline 19930118 & 7.1 & 406 & 786 & 0.5 \\
\hline 19931217 & 5.4 & 362 & 750 & 0.5 \\
\hline 19950703 & 3.5 & 448 & 649 & 1.0 \\
\hline 19960112 & 11.3 & 215 & 480 & 1.0 \\
\hline 19960131 & 8.0 & 292 & 521 & 1.6 \\
\hline 19960620 & 7.8 & 321 & 548 & 1.0 \\
\hline 19960709 & 6.3 & 404 & 631 & 1.0 \\
\hline 19970201 & 3.7 & 470 & - & 1.0 \\
\hline \multicolumn{5}{|c|}{ A1118-617 / Hen3-640 } \\
\hline 19930303 & 70.0 & 106 & 384 & 0.5 \\
\hline 19930622 & 68.0 & 106 & 407 & 0.6 \\
\hline 19940307 & 62.5 & 100 & 398 & 0.5 \\
\hline 19940703 & 62.0 & 59 & 416 & 0.5 \\
\hline 19950225 & 65.0 & 59 & 384 & 0.4 \\
\hline 19960403 & 62.0 & 82 & 398 & 0.5 \\
\hline 19970620 & 69.0 & 65 & 384 & 0.4 \\
\hline \multicolumn{5}{|c|}{ RX J0812.4-3114 / LS 992} \\
\hline 19980203 & 17.0 & 143 & 457 & 0.4 \\
\hline 19990109 & 20.5 & 458 & 457 & 0.4 \\
\hline \multicolumn{5}{|c|}{ 4U0728-25 } \\
\hline 19901227 & 9.0 & - & 210 & 0.5 \\
\hline 19910127 & 7.3 & - & 293 & 1.0 \\
\hline 19921113 & 5.0 & 176 & 361 & 0.8 \\
\hline 19930304 & 5.6 & - & 306 & 0.4 \\
\hline 19930624 & 6.0 & 115 & 192 & 0.5 \\
\hline 19931205 & 5.6 & - & 388 & 0.8 \\
\hline
\end{tabular}

know there is no difference between the disk structure of the Be stars with different spectral types, so we will use all the data from the papers referred to above.

The $\mathrm{H} \alpha$ parameters corresponding to the Be/X-ray binaries were taken from the Southampton/Valencia/SAAO data base of optical and IR observations (Reig et al. 1997). This data base covers more than 10 years of observations from a variety of telescopes in both the northern and southern hemispheres. Additional observations were made from the Bulgarian National Astronomical Observatory "Rozhen" with the Coudé spectrograph of the $2.0 \mathrm{~m} \mathrm{RCC}$ telescope giving a dispersion of $0.2 \AA \mathrm{pix}^{-1}$. The results of our measurements are summarized in Tables 1 and 2 . Our intention was to measure the $E W(\mathrm{H} \alpha)$ as defined by Dachs et al. (1981). Because traces of residual wings due to photospheric absorption were not detected, the interpolated continuum was used as baseline during the measurements. 
Table 2. $\mathrm{H} \alpha$ line parameters measured on Rozhen spectra

\begin{tabular}{|c|c|c|c|c|}
\hline $\begin{array}{c}\text { Date } \\
\text { (yyyymmdd) }\end{array}$ & $\begin{array}{c}-E W(\mathrm{H} \alpha) \\
(\AA)\end{array}$ & $\begin{array}{c}\Delta V \\
\left(\mathrm{~km} \mathrm{~s}^{-1}\right)\end{array}$ & $\begin{array}{l}F W H M \\
\left(\mathrm{~km} \mathrm{~s}^{-1}\right)\end{array}$ & $\begin{array}{c}\text { Disp. } \\
\AA_{\text {pix }}^{-1}\end{array}$ \\
\hline \multicolumn{5}{|c|}{ XPer / HD 24534} \\
\hline 19920903 & 1.9 & 382 & 526 & 0.1 \\
\hline 19920905 & 2.2 & 386 & 513 & 0.1 \\
\hline 19970817 & 10.5 & 97 & 211 & 0.2 \\
\hline $19980209 a$ & 12.3 & 133 & 287 & 0.2 \\
\hline 19980209b & 12.2 & 134 & 284 & 0.2 \\
\hline $19980219 a$ & 12.1 & 118 & 286 & 0.2 \\
\hline $19980219 b$ & 11.7 & 127 & 286 & 0.2 \\
\hline $19981102 \mathrm{a}$ & 9.8 & 142 & 251 & 0.2 \\
\hline 19981102b & 9.9 & 145 & 250 & 0.2 \\
\hline 19990309a & 6.7 & 161 & 283 & 0.2 \\
\hline $19990309 b$ & 6.3 & 164 & 285 & 0.2 \\
\hline 19990919a & 8.1 & 146 & 281 & 0.2 \\
\hline 19990919b & 7.7 & 148 & 282 & 0.2 \\
\hline \multicolumn{5}{|c|}{$\mathrm{LS} \mathrm{I}+61^{\circ} 303 / \mathrm{V} 615$ Cas } \\
\hline 19980930 & 13.3 & 293 & 607 & 0.2 \\
\hline 19980930 & 13.4 & 298 & 604 & 0.2 \\
\hline 19981001 & 11.9 & 314 & 600 & 0.2 \\
\hline 19981001 & 11.0 & 298 & 617 & 0.2 \\
\hline 19981004 & 12.3 & 346 & 554 & 0.2 \\
\hline 19981004 & 12.6 & 345 & 569 & 0.2 \\
\hline 19981009 & 10.2 & 330 & 615 & 0.2 \\
\hline 19981209 & 9.3 & 304 & 583 & 0.2 \\
\hline 19990105 & 9.3 & 339 & 590 & 0.2 \\
\hline 19990106 & 10.0 & 337 & 585 & 0.2 \\
\hline 19990306 & 10.0 & 337 & 629 & 0.2 \\
\hline 19990326 & 9.8 & 327 & 605 & 0.2 \\
\hline 19990917 & 10.7 & 368 & 624 & 0.2 \\
\hline 19990917 & 10.6 & 353 & 628 & 0.2 \\
\hline 19990919 & 9.0 & 347 & 602 & 0.2 \\
\hline 19990925 & 9.6 & 340 & 545 & 0.2 \\
\hline 19990926 & 10.4 & 327 & 527 & 0.2 \\
\hline 19991025 & 12.0 & 319 & 535 & 0.2 \\
\hline 19991126 & 10.7 & 325 & 626 & 0.2 \\
\hline
\end{tabular}

In the case of double peak profiles the parameter FWHM is measured only when the both peaks have an intensity above the half-maximum. This is because the parameter is used to investigate the broadening in the disk and it would give unusual values if measured using only one peak, i.e. it would be sensitive to the broadening process from half of the disk only. The last column in the Tables 1 and 2 represents the reciprocal dispersion of the spectra in $\AA$ per pixel.

Additionally to these observations we used the data for 4U 1145-619 from Cook \& Warwick (1987), LS I+61³03 by Zamanov et al. (1999), and LS I+61 235 by Reig et al. (2000a). The values used for the projected rotational velocity, $v \sin i$, are summarized in Table 3.
Table 3. Values of $v \sin i$ for the Be/X-ray binaries

\begin{tabular}{lcl}
\hline Object & $\begin{array}{c}v \sin i \\
\left(\mathrm{~km} \mathrm{~s}^{-1}\right)\end{array}$ & Reference \\
\hline X Per & 215 & Lyubimkov et al. (1997) \\
LS I+61 ${ }^{\circ} 303$ & 360 & Hutchings \& Crampton (1981a) \\
LS I+61 235 & 200 & Reig et al. (1997) \\
4U 1145-619 & 270 & van Paradijs (1995) \\
A 0535+26 & 254 & Clark et al. (1998) \\
V 0332+53 & $145^{*}$ & Negueruela et al. (1999) \\
4U 0115+63 & 365 & Hutchings \& Crampton (1981b) \\
A 1118-617 & 300 & Janot-Pacheco et al. (1981) \\
RX J0812-31 & 240 & Reig et al. (2000b) \\
4U 0728-25 & 200 & Corbet \& Mason (1984) \\
\hline
\end{tabular}

$\left(^{*}\right)$ Average value on base of HeI/HeII absorption lines estimates only.

\section{Comparison of the $\mathrm{H} \alpha$ line parameters for $\mathrm{Be}$ and $\mathrm{Be} / \mathrm{X}$-ray stars}

In this section we present the behaviour of the $\mathrm{H} \alpha$ parameters, one versus another, for isolated Be stars and $\mathrm{Be} / \mathrm{X}$-ray binaries.

In an attempt to provide a quantitative approach to our study, we have performed two dimensional Kolmogorov-Smirnov tests (Peacock 1983; Press et al. 1994) on every figure. This test gives us the probability that both samples are compatible with each other. Usually if the indicated probability is $<0.05$ the two data sets are statistically different. The Be stars atlases contain a lot of stars, each one observed one or several times. However for Be/X-ray binaries, we have merely 10 objects observed a lot of times. Therefore in the KS-test we used only $2-4$ points for every Be/X-ray binary, in order to have a similar sample from each group. Thus the effective number of the data points is $\gtrsim 20$, which should give sufficient accuracy for the test.

It deserves to be noted that in general the type of the profile (double or single peak) and the $V / R$ ratio can influence the positions in the diagrams, e.g. Dachs et al. (1992) detected a relationship between the $V / R$ ratio and the radial velocities of the peaks. Our attempts to use only single peak profiles or only almost symmetrical profiles $(V / R \sim 1.0 \pm 0.2)$ do not give different pictures. So in the following we will use all data independently of the $\mathrm{H} \alpha$ profile type.

\subsection{The $H \alpha$ parameters versus $v \sin i$}

Figure 1a shows the behaviour of the $\mathrm{H} \alpha$ full width at half maximum $(F W H M)$. It is well known that the width of the $\mathrm{H} \alpha$ emission line increases linearly with $v \sin i$ (Struve 1931; Andrillat \& Fehrenbach 1982; Dachs et al. 1986).

The observed points for Be/X-ray binaries are well inside the scatter of other Be stars. The KS test gives a significant probability of $0.10-0.15$, that the points are extracted from the same distribution. 
The observed correlation between stellar rotational velocities, $v \sin i$, of the Be stars and $F W H M$ of their emission-line profiles gives the strongest evidence for a rotationally supported disk-like circumstellar envelope and a coupling of photospheric and envelope rotation (e.g. Dachs et al. 1986; Hanuschik 1989). The fact that the Be/X-ray binaries have a similar distribution in Fig. 1a points that, independently of the presence of compact companion, their envelopes are axisymmetric and rotationally supported, as could be expected.

In Figs. 1b and 1c, we plot the behaviour of the $E W$ and the peak separation, $\Delta V$, versus $v \sin i$, respectively. In Fig. 1b the KS test gives a probability of $0.01-0.001$, which suggests strongly that both samples are statistically different. Our numerical experiments suggest that this is mainly due to most Be/X-ray binaries not achieving $E W(\mathrm{H} \alpha)$ values above $25 \AA$. Some influence on the KS-test results has also the fact that low values of $v \sin i$ are missing among the observed Be/X-ray binaries, but this has minor contribution. Therefore, Fig. 1b supports the original result by Reig et al. (1997) that the Be/X-ray binaries have, on average, a lower $E W(\mathrm{H} \alpha)$ when compared to a set of isolated Be stars.

In Fig. 1c the KS test gives a probability of $0.2-0.3$, which indicates that the behaviour of the peak separation for both types is very similar. It is worth noting that (although it is not statistically significant), the peak separation of the Be/X-ray binaries does not achieve values smaller than the peak separation of the Be stars $(\Delta V$ is a measure of the disk size, see Eq. (2)).

\subsection{The $H_{\alpha}$ width parameters versus the equivalent width}

In Fig. 2 the $\mathrm{H} \alpha$ width parameters $\Delta V$ and $F W H M$, normalized to $v \sin i$, have been plotted as a function of the $E W(\mathrm{H} \alpha)$. In the top panel, it is clear that the $\mathrm{Be} / \mathrm{X}$ ray data points as well as their linear fit are considerably shifted relative to the behaviour of the Be stars. The KS test gives a probability of $\sim 0.001$ if we use all data points, and probability $\sim 0.01$ if we use only data with $E W \leq 20 \AA$. This fact can be interpreted as indicating the presence of denser disks and will be discussed in Sect. 4 .

In the bottom panel, the KS test for $E W \leq 20 \AA$ gives a probability of $0.20-0.40$. It is also obvious that both samples occupy one and the same place at low $E W$. This result is not unexpected and implies that the processes relevant to the line broadening (Kepler rotation, thermal broadening, non-coherent scattering) are identical in the disks of the $\mathrm{Be}$ and $\mathrm{Be} / \mathrm{X}$-ray stars, when the latter are non perturbed. There are several cases when one of the wings is strongly affected, and, as a result, it has an intensity below the half maximum. Probably this is an effect of global one-armed oscillations (see Negueruela et al. 1998, for a discussion of this effect). In such cases we did not measure the $F W H M$ for the reasons mentioned in Sect. 2.

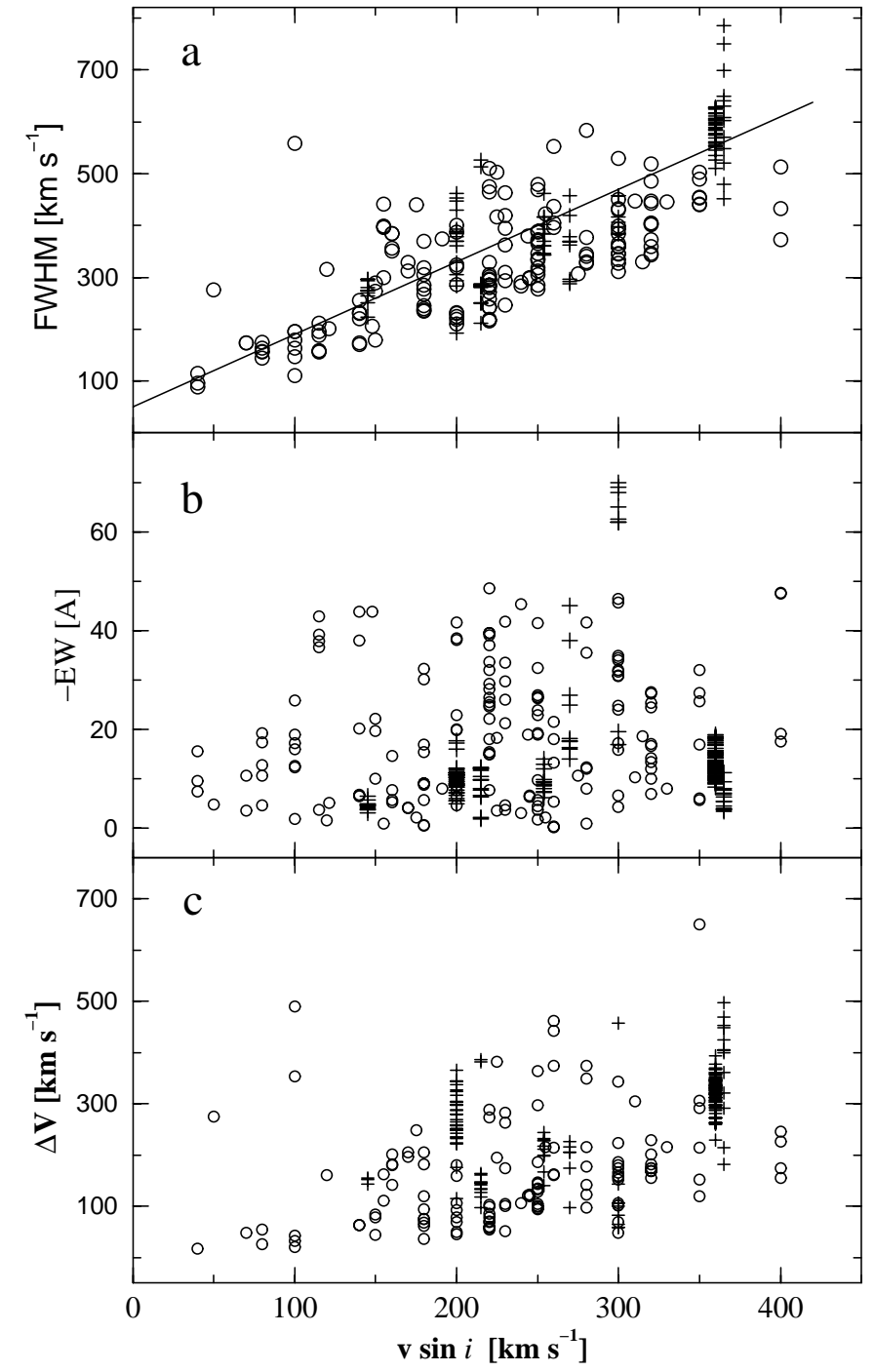

Fig. 1. a-c) The $\mathrm{H} \alpha$ line parameters versus $v \sin i$. The circles represent the Be stars, the crosses - the Be/X-ray binaries; a) $F W H M$ versus $v \sin i$. The solid line is $F W H M=1.4 v \sin i+$ $50 \mathrm{~km} \mathrm{~s}^{-1}$, which is the average behaviour of the Be stars, as defined by Hanuschik (1989); b) $E W(\mathrm{H} \alpha)$ versus $v \sin i$; c) The distance between the peaks versus $v \sin i$

\subsection{FWHM versus $\Delta V$}

In Fig. 3 we plot the $F W H M$ against the peak separation, both normalized to $v \sin i$. The KS test gives here a probability of about 0.001 . This strongly indicates that the two samples are drawn from different distributions.

The FWHM of the $\mathrm{H} \alpha$ emission-line profiles are essentially determined by the effective rotational velocity of those regions of the envelope which are emitting an important fraction of the $\mathrm{H} \alpha$ radiation. In Fig. 3 the crosses of the Be/X-ray binaries are shifted towards high $\Delta V$. This implies that, at the same effective emitting size, their disks are smaller. Such a result, can be interpreted as a clear indication that in many cases the outer parts of the Be/Xray disks are missing, probably truncated by the neutron star. 


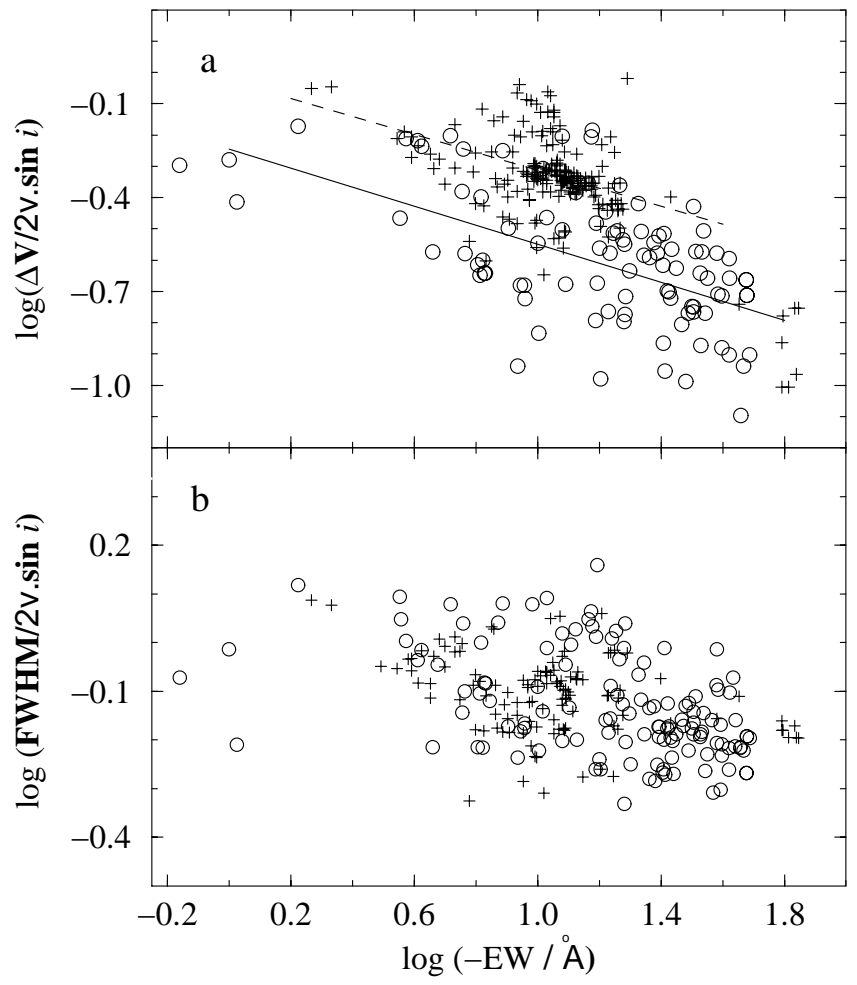

Fig. 2. a) and b) The $\mathrm{H} \alpha$ line parameters versus $E W(\mathrm{H} \alpha)$. a) Plot of $\log (\Delta V /(2 v \sin i))$ versus $E W(\mathrm{H} \alpha)$. The lines represent the best linear fits: the solid line over the circles (Be stars), the dashed line over the crosses (Be/X-ray stars). The best fit line of the Be/X-ray binaries is shifted to denser circumstellar disks; b) $\log (F W H M /(2 v \sin i))$ versus $\log E W(\mathrm{H} \alpha)$. The Be/X-ray binaries and isolated Be stars mixed together

\section{Dense circumstellar disks in the $\mathrm{Be} / \mathrm{X}$-ray binaries}

It is known that the $\mathrm{H} \alpha$ peak separation and the equivalent width correlate for the Be stars (Hanuschik et al. 1988). Hanuschik (1989) have derived the law

$\log \left(\frac{\Delta V}{2 v \sin i}\right)=-a \log \left(\frac{-E W(\mathrm{H} \alpha)}{\AA}\right)+b$,

where the average values are $a \simeq 0.32$ and $b \simeq-0.2$. It is worth noting that when using almost twice as many points we obtained practically the same values $a=0.34 \pm 0.06$ and $b=-0.18 \pm 0.07$. The law in Eq. (1) expresses that the outer radius grows as the $E W(\mathrm{H} \alpha)$ becomes larger, because for rotationally dominated profiles the peak separation can be regarded as a measure of the outer radius of the $\mathrm{H} \alpha$ emitting disk (Huang 1972):

$$
\left(\frac{\Delta V}{2 v \sin i}\right)=\left(\frac{R_{\text {disk }}}{R_{*}}\right)^{-j},
$$

with $j=0.5$ for Keplerian rotation and $j=1$ for conservation of angular momentum.

The same quantities for the Be/X-ray binaries are plotted in Fig. 4. The dashed line represent the average behaviour of Be stars as defined by Hanuschik (1989).

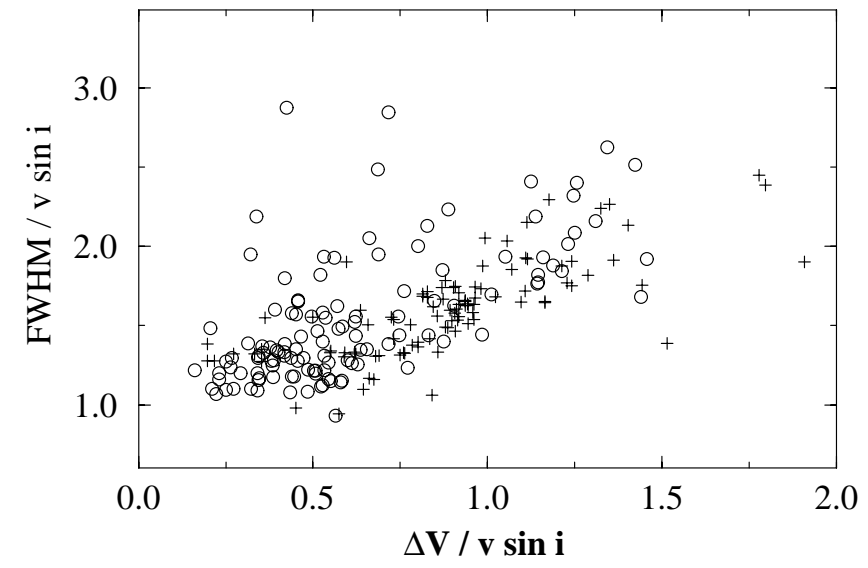

Fig. 3. $F W H M$ versus the peak separation, $\Delta V$, normalized with $v \sin i$. The symbols are the same as in Fig. 1

Hanuschik et al. (1988) consider that the different electron density $\left(N_{\mathrm{e}}\right)$ is the factor responsible for the vertical scattering in this figure. They estimated that the limits for the Be stars correspond to

$N_{\mathrm{e}, \max } / N_{\mathrm{e}, \min } \simeq 4.5$.

From Fig. 4 it can be seen that almost all the data points for the $\mathrm{Be} / \mathrm{X}$-ray binaries are above the average line, i.e. they are shifted towards the denser disks. This indicates that the circumstellar disks in the Be/X-ray systems are about $\simeq 2$ times more dense than disks in isolated Be stars.

This result is very unlikely to be due to the lower resolution of the observations involving Be/X-ray binaries, because at least for three systems, X Per, LS I+61 303 and LS I+61 235 , some observations had high spectral resolution (0.10-0.067 $\left.\AA \mathrm{pix}^{-1}\right)$ and these data do not show significant differences with respect to the lower resolution observations.

This behaviour was first discovered for LS I+61 303 (Zamanov et al. 1999) and for LS I+61235 (Reig et al. 2000a). Given the large amount of data collected for the

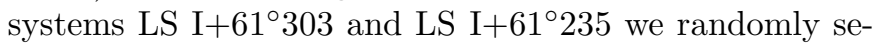
lected a subsample of their observations in order not to give too much weight to them in Fig. 4.

The possibility that the disks in the Be/X-ray binaries are denser has already been suggested by Negueruela et al. (1998) in connection with the shorter periods of $V / R$ variability.

\section{Discussion}

The Balmer emission lines that characterize the spectra of Be stars provide information about the physical conditions of the circumstellar equatorial disk. In this work we have used the $\mathrm{H} \alpha$ emission line spectral parameters to compare the properties of the disks in isolated Be stars and Be stars in Be/X-ray binaries. Our goal is to assess the possible effect of the compact object on the Be star's disk.

In our comparative study, we find similarities but also interesting differences in the physical properties of both type of systems. 


\subsection{Similarities}

The similarities seem to be related to the overall structure of the disks, as could be expected. Firstly, the disks in Be/X-ray binaries, like those in isolated Be stars, are axisymmetric and rotationally supported. Secondly, isolated Be stars and Be stars in binaries populate the same place in the $F W H M-E W$ diagram for low values of the $E W$. In other words, the line broadening processes (such as Keplerian rotation, thermal broadening, non-coherent scattering) are similar. Another similarity was presented by Telting (2000). He pointed out that the disk growth rates after a disk-loss episode for stars of similar spectral type are similar: $410^{-9} M_{\odot} \mathrm{yr}^{-1}$ for the Be/X-ray binary X Per (Telting et al. 1998) and $3.810^{-9} M_{\odot} \mathrm{yr}^{-1}$ for $\mu$ Cen (Hanuschik et al. 1993).

\subsection{Differences}

The differences begin to emerge when the disk develops. The results of Reig et al. (1997) as well as the KS-test performed in Fig. $1 \mathrm{~b}$ point to that the $\mathrm{Be} / \mathrm{X}$ ray binaries show smaller $E W$ than isolated Be stars. Only the systems $4 \mathrm{U} 1145-619$ and A 1118-617 (located at $v \sin i=270 \mathrm{~km} \mathrm{~s}^{-1}$ in Fig. 1) and A1118-617 (at $v \sin i=300 \mathrm{~km} \mathrm{~s}^{-1}$ ) show $E W$ comparable to those of isolated Be stars. The orbital period of $4 \mathrm{U} 1145-619$ is $188 \mathrm{~d}$ and that of A $1118-617$ is unknown but likely to be of the order of hundreds of days according to Corbet's diagram (Corbet 1986). With such wide orbits we would not expect the neutron star to have a strong impact on the disk evolution. In relation with this result there is the possibility that the peak separation $\Delta V$ in Be/X-ray systems tends to be larger than in Be stars (Fig. 1c). Although this finding could be due, in general, to the lower resolution data for $\mathrm{Be} / \mathrm{X}$-ray binaries, if real, it can represent further evidence supporting the idea that the Be stars in $\mathrm{X}$-ray binaries cannot develop extended disks because of its interaction with the neutron star (provided that $\Delta V$ can be considered as a measure of the disk radius).

The strongest evidence for a different behaviour of the circumstellar disks in $\mathrm{Be}$ and $\mathrm{Be} / \mathrm{X}$-ray systems is provided by Figs. $2 \mathrm{a}$ and 4 . In the $\Delta V-E W$ diagram, Be/Xray binaries are located in a higher region than isolated Be stars, indicating that they are, on average, about two times denser. Negueruela et al. (1998) noticed that the quasi periods of the $V / R$ variability observed in $\mathrm{Be} / \mathrm{X}$ ray binaries are, on average, much shorter than those of isolated Be stars. They suppose that this difference may be caused by denser envelopes in the binary systems. Our results seem to confirm such idea. Given the tendency of Be/X-ray binaries to occupy a preferable position in this diagram it would be very interesting if we were able to find a correlation between the position of the star in this diagram and other parameters, such as the Balmer decrement, the period of $V / R$ variability, the temperature, the rotational velocity, the chemical abundance, etc.

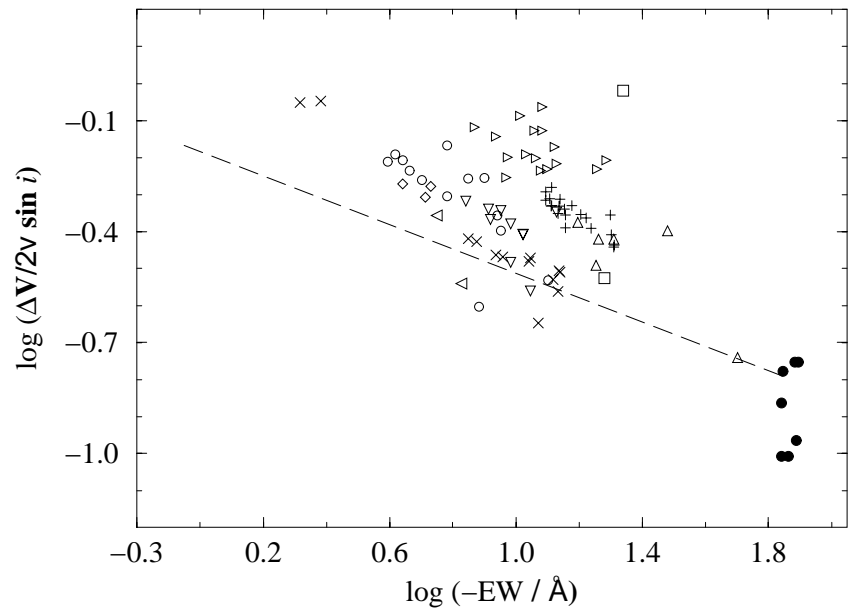

Fig. 4. Plot of $\log (\Delta V / 2 v \sin i)$ versus $E W(\mathrm{H} \alpha)$ of Be/X-ray binaries. The dashed line represents the average behaviour of the Be stars (from Hanuschik 1989). It is apparent that the data points for the Be/X-ray binaries are shifted up, towards

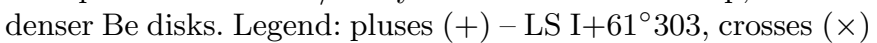
- X Per, diamonds - BQ Cam, triangles up - 4U 1145-619, triangles down - A $0535+26$, triangles right $-\mathrm{LS} \mathrm{I}+61^{\circ} 235$, triangles left - 4U 0728-25, open circles - V635 Cas, filled circles - A 1118-617, squares - LS 992

One possible explanation for the above mentioned differences is truncation of the Be star's disk by the compact companion. The continuous revolution of the neutron star around the Be star primary prevents the formation of an extended disk in Be/X-ray binaries. This is expected to occur for short orbital period systems. As a result of its gravitational field, the compact object not only will accrete a part of the disk material, but will distort the velocity of the particles at the outer disk. Probably a part of the material can be pushed in the inner part of the disk. This seems quite realistic especially if the neutron star acts as propeller or ejector, as it was supposed for LS I+61 303 (Zamanov 1995).

For systems with longer orbital periods ( it seems unlikely that the neutron star has any influence on the Be star disk. In these systems the denser disks might be a selection effect due to the fact that the neutron star will act as X-ray pulsar only if the disk is dense. A neutron star orbiting at long distance around a Be star with low density disk will act as propeller (accretion onto the magnetosphere) or ejector (radio pulsar). If this is the case, a careful analysis of photospheric conditions of the long period $\mathrm{Be} / \mathrm{X}$-ray stars can provide us with valuable information on this issue.

The idea of disk truncation has been put on stronger theoretical grounds by Negueruela et al. (2000), who interpreted the X-ray and optical characteristics of the Be/X-ray system $4 \mathrm{U} 0115+63 / \mathrm{V} 635$ Cas as warping/tilting episodes of the disk. One of the best candidates to produce the high optically thick disks needed for radiation-driven warping is disk truncation.

Finally, while the compact companion does not seem to affect the formation and initial building-up stages of 
the circumstellar disk in Be/X-ray systems, it does seem to affect its subsequent characteristics and development.

\section{Conclusions and open questions}

From our comparative study between the $\mathrm{H} \alpha$ emission lines of Be/X-ray binaries and those of isolated Be stars we find that:

- The envelops in the Be/X-ray binaries are axisymmetric and rotationally supported as in isolated Be stars;

- The line broadening is similar in the isolated Be stars and the Be/X-ray binaries;

- The circumstellar disks in Be/X-ray binaries are, on average, two times denser than those in isolated Be stars;

- The relationships between the various $\mathrm{H} \alpha$ parameters ( $F W H M, \Delta V, E W$ ) seem to indicate the presence of smaller and denser disks in Be/X-ray binaries than in isolated Be stars, most likely truncated by the compact object.

Some of the open questions which should be addressed in the future are:

- What is the behaviour of the other emission lines in Be/X-ray binaries? The relative intensities of Balmer lines (Balmer decrement) can give more information about the temperature and electron density of the disk. The optically thin lines of FeII also can give us important information.

- What is the behaviour of the Be binary systems where the companion is not a neutron star?

- Do the physical properties of the optical companion (metallicity, instability in the photosphere induced by binary rotation, etc.) contribute to producing denser disks?

Acknowledgements. We are very grateful to the referee Dr. R. Hanuschik for us useful comments and suggestions. RZ acknowledges support from Direccion general de relaciones culturales y cientificas, Spain. JM acknowledge partial support by DGESIC (PB97-0903 and PB98-0670-C0201) and by Junta de Andalucía (Spain). P. Reig acknowledges support via the European Union Training and Mobility of Researchers Network Grant ERBFMRX/CT98/0195.

\section{References}

Corbet, R. H. D. 1986, MNRAS, 220, 1047

Andrillat, Y., \& Fehrenbach, Ch. 1982, A\&AS, 48, 93

Andrillat, Y. 1983, A\&AS, 53, 319

Bjorkman, J. E., \& Cassineli, J. P. 1993, ApJ, 409, 429

Clark, J. S., Tarasov, A. E., Steele, I. A., et al. 1998, MNRAS, 294,165

Cook, M. C., \& Warwick, R. S. 1987, MNRAS, 227, 661
Corbet, R. H. D., \& Mason, K. O. 1984, A\&A, 131, 385

Dachs, J., Eichendorf, W., Schleicher, H., et al. 1981, A\&AS, 43, 427

Dachs, J., Hanuschik, R., Kaiser, D., et al. 1986, A\&AS, 63, 87

Dachs, J., Hummel, W., \& Hanuschik, R. W. 1992, A\&AS, 95, 437

Hanuschik, R. W. 1986, A\&A, 166, 185

Hanuschik, R. W. 1989, Ap\&SS, 161, 63

Hanuschik, R. W., Kozok, J. R., \& Kaiser, D. 1988, A\&A, 189, 147

Hanuschik, R. W., Dachs, J., Baudzus, M., \& Thimm, G. 1993, A\&A, 274, 356

Hoffleit, D., \& Jascheck, C. 1982, The Bright Star Catalogue, 4th Edition, Yale University

Huang, S.-S. 1972, ApJ, 171, 549

Hutchings, J. B., \& Crampton, D. 1981a, PASP, 93, 486

Hutchings, J. B., \& Crampton, D. 1981b, ApJ, 247, 222

Janot-Pacheco, E., Ilovaisky, S. A., \& Chevalier, C. 1981, A\&A, 99, 274

Lyubimkov, L. S., Rostopchin, S. I., Roche, P., \& Tarasov, A. E. 1997, MNRAS, 286, 549

Negueruela, I. 1998, A\&A, 338, 505

Negueruela, I., Reig, P., Coe, M. J., \& Fabregat, J. 1998, A\&A, 336,251

Negueruela, I., Roche, P., Fabregat, J., \& Coe, M. J. 1999, MNRAS, 307, 695

Negueruela, I., Okazaki, A. T., Fabregat, J., et al. 2000, A\&A, submitted

Peacock, J. A. 1983, MNRAS, 202, 615

Press, W., Teukolsky, S. A., Veterling, W. T., \& Flannery, B. P., 1993, Numerical Recipes (Cambridge University Press)

Reig, P., Fabregat, J., \& Coe, M. J. 1997, A\&A, 322, 193

Reig, P., Coe, M. J., Stevens, J. B., et al. 1997, in The Transparent Universe, Proceedings of the 2nd INTEGRAL Workshop, ESA SP-382

Reig, P., Negueruela, I., Coe, M. J., et al. 2000a, MNRAS, 317, 205

Reig, P., Negueruela, I., Buckley, D. A. H., et al. 2000b, A\&A, in press

Roche, P., Coe, M. J., Fabregat, J., et al. 1993, A\&A, 270, 122

Slettebak, A., Collins II, G. W., \& Truax, R. 1992, ApJS, 81, 335 (SCT92)

Stevens, J. B., Reig, P., Coe, M. J., et al. 1997, A\&A, 322, 183

Struve, O. 1931, ApJ, 73, 94

Unger, S. J., Roche, P., Negueruela, I., et al. 1998, A\&A, 336, 960

Telting, J. H., Waters, L. B. F. M., Roche, P., et al. 1998, MNRAS, 296, 785

Telting, J. H. 2000, in Proc. IAU Coll. 175, The Be Phenomenon in Early Type Stars, ed. M. A. Smith, H. F. Henrichs, \& J. Fabregat, ASP Conf. Ser., in press

Van Paradijs, J. 1995, in X-Ray Binaries, ed. W. Lewin, J. van Paradijs, \& E. P. J. van den Heuvel (Cambridge University Press), 536

Zamanov, R. 1995, MNRAS, 272, 308

Zamanov, R., Martí, J., Paredes, J. M., et al. 1999, A\&A, 351, 543 